\title{
CLÍNICA, ELETROCARDIOGRAFIA E ANÁLISE LABORATORIAL DE GATOS TRATADOS COM LEVAMISOL
}

\section{Clinic, electrocardiography and laboratorial analysis of cats treated with levamisol}

\author{
MELCHERT, A. ${ }^{1}$; NOGUEIRA, R.M.B. ${ }^{1}$; LAPOSY, C.B. ${ }^{1}$; BARRACAR, K.C. ${ }^{2}$; \\ FERREIRA, F.L. ${ }^{2}$
}

\author{
1 Professor, Universidade do Oeste Paulista (Unoeste), Presidente Prudente, SP. \\ ${ }^{2}$ Acadêmica de Medicina Veterinária, Unoeste. \\ Endereço para correspondência: Alessandra Melchert: alessandravet@unoeste.br
}

\section{RESUMO}

O levamisol pode ser usado como parasiticida e indutor de imunidade. Na literatura há conflitos quanto à dose, vias de administração, efeitos adversos e toxicidade do fármaco em gatos. Com o objetivo de avaliar os efeitos clínicos, laboratoriais e eletrocardiográficos do levamisol em gatos, foram estudados nove animais, utilizando-se a dose imunomoduladora de $25 \mathrm{mg} / \mathrm{gato}$, via oral, em dias alternados, por 3 aplicações (dias 1, 3 e 5). Os animais foram avaliados antes do levamisol (controle), 60, 180 e 360 minutos após administração do levamisol, em todos os dias de tratamento. Avaliou-se a temperatura (T), frequência respiratória $(f)$, frequência cardíaca (FC), pressão arterial sistólica (PAS) e eletrocardiograma (ECG) em todos os momentos citados. Hemograma, função hepática, incluindo alanino aminotransferase (ALT) e gamaglutamil transferase (GGT), proteínas totais, albumina e globulinas foram avaliados antes e após a última aplicação do levamisol. Não foram observadas alterações da $f, A L T$, GGT e proteínas. A T, FC e PAS elevaram-se significativamente em diferentes momentos em relação ao controle, permanecendo, no entanto, dentro dos limites fisiológicos para a espécie. Sialorréia e vômito foram observados em $33,3 \%$ e $11,1 \%$ dos animais, respectivamente. No ECG foi observado bloqueio átrio-ventricular de $1^{\circ}$ e $2^{\circ}$ grau em $22,2 \%$ dos animais, nos dias 1 e 5, e complexos atriais prematuros em 33,3\%, nos dias 1 e 3 . No hemograma houve diminuição do número de linfócitos após a terceira dose administrada do fármaco. Conclui-se que na dose estudada o levamisol provoca efeitos tóxicos em gatos e deve ser usado com cautela.

Palavras-chave: efeitos tóxicos; felino; imunomodulador

\begin{abstract}
The levamisole is an antihelminthic drug that has been used as an immunomodulator. However, there are some doubts regarding the dosage, administration route, side effects and toxicity of the drug in cats. In order to evaluate the effects of levamisole on clinical, laboratorial and electrocardiogram (ECG) of cats, nine animals were orally treated with three applications of levamisole in immunomodulatory dose $(25 \mathrm{mg} / \mathrm{per}$ cat), in intervals of 48 hours. The animals were examined before treatment (control) and after 60,180 and 360 minutes after administration of drug. Temperature, respiratory rate $(f)$, heart rate $(\mathrm{HR})$, systolic arterial pressure (SAP), and ECG were the parameters evaluated. Hemogram, alanine aminotransferase (ALT) and $\gamma$-glutamyltransferase (GGT), total serum protein, albumin and globulin were carried out in order to evaluate the hepatic function before and after the last levamisole application. Alterations of $f$, ALT, GGT and proteins were not observed. Temperature, HR and SAP increased significantly in different moments after treatment, however the values were inside of the physiologic range. It was observed a decrease of lymphocytes number after the levamisole administration. Sialorrhea and vomiting were observed in $33.3 \%$ and $11.1 \%$ of the animals, respectively. ECG revealed first and second degree atrium-ventricular blockade in $22.2 \%$ and atrial premature complexes was observed in $33.3 \%$ of the cats. In conclusion, levamisole given orally at 25 $\mathrm{mg} /$ per cat causes side effects and be used carefully.
\end{abstract}

Key words: feline; immunomodulator; side effects 


\section{INTRODUÇÃO}

O levamisol é um antiparasitário do grupo dos imidazotiazóis, que atua em receptores colinérgicos, acumulando na fenda sináptica e causando hiperexcitabilidade e paralisia espástica de nematódeos. Também, é capaz de induzir resposta imune celular nos animais (Almeida e Aires, 2006), estimulando a diferenciação de células $T$ e a resposta aos antígenos em animais com depressão da função de células $T$, com pouco ou sem efeito no sistema imune perfeito (Canal et al., 2004).

O produto apresenta toxicidade para algumas espécies animais como cavalos, cães e gatos (Almeida e Aires, 2006), entretanto a literatura é conflitante. Andrade e Santarém (2002) relatam que a dose tóxica é próxima da terapêutica, enquanto Booth e McDonald (1992) relatam que se trata de um vermífugo seguro, com margem de segurança entre 2 a 5 vezes superior à dose eficaz. As doses recomendadas para gatos variam na literatura. Booth e McDonald (1992) indicam o levamisol como antiparasitário na dose de 5 a $11 \mathrm{mg} / \mathrm{kg}$ para cães e gatos, enquanto Andrade e Santarém (2002) indicam-no na dosagem de 20 a 40 $\mathrm{mg} / \mathrm{kg}$, a cada 6 horas, por 5 a 6 tratamentos, na terapia de vermes pulmonares. Como imunoestimulante, as doses variam de $25 \mathrm{mg} / \mathrm{gato}$, a cada 48 horas, por 3 aplicações (Rochett, 2001), enquanto Allen et al. (2005) indicam três administrações na dose de $5 \mathrm{mg} / \mathrm{kg}$, distribuídas em uma semana.

Os cavalos e carnívoros são mais susceptíveis às intoxicações por levamisol, com sinais semelhantes àqueles produzidos por organofosforados (Baldani et al., 1999). Em cães, pode desencadear reações farmacodérmicas (Sousa et al., 2005), eritema multiforme e necrólise epidérmica tóxica (Scott et al., 2001).
Tendo em vista a variabilidade das doses de levamisol recomendadas pela literatura e a falta de estudos que avaliem a toxicidade do fármaco em gatos, o presente estudo teve por objetivo avaliar clinica e laboratorialmente gatos tratados com levamisol, por via oral.

\section{MATERIAL E MÉTODOS}

Foram utilizados nove gatos, sem raça definida (SRD), adultos, machos e fêmeas, com peso corporal entre 3 e $5 \mathrm{~kg}$, hígidos, selecionados por meio da normalidade dos exames físico e laboratoriais.

Os gatos foram submetidos à administração de levamisol como imunomodulador, por via oral, na dose de $25 \mathrm{mg} /$ gato, a cada 2 dias, por três aplicações (Dia 1, Dia 3 e Dia 5) (Rochett, 2001). Foram avaliados a temperatura corporal $(T)$, frequência respiratória $(f)$, frequência cardíaca (FC), eletrocardiograma (ECG), pressão arterial sistólica (PAS) não invasiva, e ocorrência de efeitos colaterais tais como vômito, diarréia, sialorréia (intensidade discreta, moderada ou acentuada) e alteração do diâmetro pupilar (midríase ou miose), nos seguintes momentos: Dia 1 (Primeira aplicação) Controle (Antes da administração do levamisol); 60 (60 min), 180 (180 min) e 360 (360 min) minutos após administração do levamisol; Dia 3 (Segunda aplicação) e Dia 5 (Terceira aplicação), nos mesmos momentos citados (Controle, $60 \mathrm{~min}, 180$ $\mathrm{min}$ e $360 \mathrm{~min}$ ).

Hemograma completo, função hepática, incluindo alanino aminotransferase (ALT) e gamaglutamil transferase (GGT), proteínas totais, albumina e globulina foram avaliados antes e após a última aplicação do levamisol, considerando-se os valores normais estabelecidos para a espécie (Kaneko et al, 1997; Jain, 1993). 
Para avaliação estatística dos dados obtidos em cada momento, foi utilizado o teste de análise de variância para amostras dependentes. Os contrastes entre as médias dos grupos foram verificados pelo teste Tukey, considerando um nível de significância de 5 \% (Morrison, 1990).

\section{RESULTADOS}

A avaliação dos resultados revelou poucas alterações nos parâmetros vitais dos animais estudados. Na avaliação da FC, no dia 5, observou-se aumento significativo da FC após 60 e 180 minutos da administração do fármaco, em relação ao controle e aos 360 minutos após administração $(P<0,05)$. Entretanto, os valores permaneceram dentro da normalidade para a espécie em todos os momentos. Na avaliação da $f$ não foram observadas diferenças significativas em nenhum dos momentos avaliados, nos dias 1 , 3 ou 5, observando-se valores fisiológicos para gatos (Tabela 1).

A PAS revelou aumento significante em 180 minutos após a administração do levamisol em relação ao controle, no dia 1 $(P<0,05)$. Na avaliação da $\mathrm{T}$, no dia 5 , observou-se aumento significante em M180, em relação ao controle e aos 360 minutos da avaliação $(P<0,05)$. Todos os valores permaneceram dentro dos limites fisiológicos para a espécie (Tabela 1).

Foi observada a ocorrência de sialorréia em três animais estudados $(33,3 \%)$. Em um gato, sialorréia de discreta intensidade ocorreu aos 360 minutos, enquanto dois gatos manifestaram a ocorrência entre 60 e 180 minutos após o levamisol, sendo uma de intensidade moderada e outra acentuada. Um dos animais $(11,1 \%)$ apresentou vômito espumoso entre 60 e 180 minutos, e um gato apresentou midríase $(11,1 \%)$ também

Tabela 1 - Valores de freqüência cardíaca, freqüência respiratória, pressão arterial sistólica e temperatura em gatos submetidos a administração oral de levamisol em esquema imunoestimulante, em diferentes momentos

\begin{tabular}{|c|c|c|c|}
\hline Parâmetro & Dia 1 & Dia 3 & Dia 5 \\
\hline \multicolumn{4}{|c|}{ Frequência Cardíaca (bpm) } \\
\hline M0 & $208,9 \pm 19,8$ & $201,8 \pm 18,8$ & $200 \pm 32$ \\
\hline M60 & $205,0 \pm 22,1$ & $201,1 \pm 25,1$ & $235,6 \pm 27,4^{1}$ \\
\hline M180 & $213,3 \pm 25,0$ & $198,9 \pm 20,2$ & $230 \pm 26,5^{1}$ \\
\hline M360 & $203 \pm 21,2$ & $205,6 \pm 22,2$ & $201,1 \pm 34,8$ \\
\hline \multicolumn{4}{|c|}{ Frequência Respiratória (mpm) } \\
\hline MO & $59,3 \pm 16$ & $56 \pm 18,5$ & $57,3 \pm 20,4$ \\
\hline M60 & $61,8 \pm 18,9$ & $58,2 \pm 19,3$ & $57,3 \pm 16,4$ \\
\hline M180 & $57,3 \pm 13,1$ & $56 \pm 22,5$ & $62,7 \pm 21,5$ \\
\hline M360 & $51,6 \pm 21,9$ & $58 \pm 16,2$ & $56,8 \pm 19,4$ \\
\hline \multicolumn{4}{|c|}{ Temperatura $(\mathrm{C})$} \\
\hline MO & $38,5 \pm 0,7$ & $38,7 \pm 0,6$ & $38,5 \pm 0,8$ \\
\hline M60 & $38,9 \pm 0,4$ & $38,7 \pm 0,7$ & $38,9 \pm 0,7$ \\
\hline M180 & $38,7 \pm 0,5$ & $38,7 \pm 0,3$ & $39,1 \pm 0,7^{1}$ \\
\hline M360 & $38,5 \pm 0,4$ & $38,5 \pm 0,4$ & $38,6 \pm 0,4$ \\
\hline \multicolumn{4}{|c|}{ Pressão arterial sistólica (mmHg) } \\
\hline M0 & $141,7 \pm 33$ & $191,1 \pm 35,5$ & $121,1 \pm 19$ \\
\hline M60 & $160 \pm 29,2$ & $186,7 \pm 40,9$ & $125 \pm 16,2$ \\
\hline M180 & $175,6 \pm 27,4^{2}$ & $180 \pm 50,7$ & $122,2 \pm 38,7$ \\
\hline M360 & $175 \pm 20,9$ & $196,7 \pm 30,8$ & $118,9 \pm 17,6$ \\
\hline
\end{tabular}

$\mathrm{P}>0,05$ em relação a $\mathrm{M0}$ e $\mathrm{M} 360 ; 2-\mathrm{p}>0,05$ em relação a $\mathrm{M0}$. 
$2 \mathrm{~cm} / \mathrm{mV} 35 \mathrm{~Hz} 60 \mathrm{~Hz}$

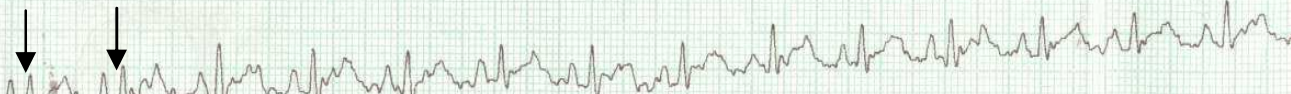

Figura 1 - Complexo atrial prematuro (APC) (ponta das setas) observado no ECG de gatos, após a administração oral de levamisol.

aos 60 e 180 minutos após administração do levamisol.

$\mathrm{Na}$ avaliação do ECG, observou-se a ocorrência de arritmias em cinco (55,5\%) gatos. No dia 1, bloqueio átrio-ventricular de $1^{\circ}$ grau (BAV I) foi observado em 1 animal (11,1\%), enquanto outro gato $(11,1 \%)$ apresentou bloqueio átrioventricular de $2^{\circ}$ grau (BAV II) no dia 5, sendo que ambas as alterações ocorreram aos 180 minutos de avaliação. Complexos atriais prematuros (APCs) foram observados em 3 gatos $(33,3 \%)$, sendo que uma ocorreu no dia 1, após 60 minutos, e as outras no dia 3 , em dois gatos diferentes, aos 180 e aos 360 após o levamisol (Figura 1).

$\mathrm{Na}$ avaliação do número de hemácias, hematócrito, hemoglobina, função hepática (ALT e GGT) e das proteínas séricas (proteína total, albumina e globulinas) não foram observadas diferenças significativas quando comparados os momentos antes e depois da administração do levamisol.

No leucograma não ocorreram diferenças estatísticas significativas nos momentos antes e depois da administração do levamisol, exceto para a contagem absoluta de linfócitos, que revelou diminuição significativa após 0 tratamento com levamisol $(P<0,05)$, com ocorrência de linfopenia absoluta em 2 gatos $(22,2 \%)$, não observada em nenhum animal antes do tratamento.

\section{DISCUSSÃO}

O levamisol é um fármaco há muito utilizado na Medicina Veterinária, tanto por sua atividade antiparasitária, quanto por seus efeitos imunomoduladores. No entanto, diversos estudos relatam a toxicidade do fármaco, ligada à inibição da colinesterase (Canal et al., 2004). Neste estudo, as alterações observadas foram tipicamente colinérgicas, e incluíram sialorréia, vômito e midríase, corroborando Allen et al. (2005), que citam estes sinais como comuns na toxicidade do levamisol em gatos. A salivação pode ocorrer por contração muscular glandular, também responsável pela midríase, e o vômito pode estar relacionado com a alta motilidade das vias digestivas (Canal et al., 2004).

As doses recomendadas pela literatura variam de $25 \mathrm{mg} / \mathrm{gato}$ (Rochett, 2001), até $40 \mathrm{mg} / \mathrm{Kg}$ (Andrade e Santarém, 2001). Para as espécies canina e felina, doses orais de até $80 \mathrm{mg} / \mathrm{kg}$ podem não ser fatais, embora levem à sinais de intoxicação. No entanto, $50 \%$ desta dose, $40 \mathrm{mg} / \mathrm{kg}$, leva-os a óbito em 10 a 15 minutos, se administrada parenteralmente (Canal et al., 2004). Neste estudo, observou-se que a baixa dose utilizada (25 mg/gato), por via oral, foi capaz de produzir sinais de toxicidade em gatos, sugerindo que a dose tóxica para esta espécie pode estar bem mais próxima da dose terapêutica.

Observou-se, nos animais estudados, diminuição significativa dos linfócitos após administração do levamisol, ao contrário do que ocorre em outras espécies, onde o fármaco produz aumento do número e da atividade dos linfócitos em animais doentes, mas com pouco ou sem efeito em animais imunocompetentes (Canal et al., 2004). 
A ausência de efeito imunoestimulante do levamisol sobre os valores de leucócitos estudados vem reforçar a hipótese de que o seu efeito estimulante seja mais evidente quando administrado a animais imunodeprimidos. Entretanto, deve-se ressaltar que após o término do tratamento, além da redução significativa dos linfócitos, $22,2 \%$ dos gatos desenvolveram um quadro de linfopenia absoluta, indicando que estudos adicionais sobre os efeitos imunoestimulantes do levamisol em gatos devem ser realizados.

$\mathrm{Na}$ avaliação da função hepática não se observou diferenças significativas em ALT e GGT em gatos, uma vez que não houve alteração da permeabilidade da membrana hepato-celular, com a liberação dessas enzimas solúveis, que normalmente ocorre em até 3 horas e meia após a injúria (Meyer e Harvey, 1998).

Relatos em cães e ratos (Nogueira et al., 2003; Onuaguluchi e Igbo, 1990) têm descrito a ocorrência de arritmias e bradicardia com 0 uso do levamisol, entretanto, não foram encontrados relatos que avaliassem a ocorrência de alterações do ritmo cardíaco em gatos sob terapia com o fármaco. Mais de $50 \%$ dos animais estudados apresentam alterações do ritmo cardíaco após a administração oral de levamisol, que surgiram entre 60 e 360 minutos após a administração do fármaco, o que sugere que gatos tratados com levamisol devem receber especial atenção em relação ao ritmo cardíaco, principalmente se houver doença cardíaca de base.

As alterações eletrocardiográficas observadas com o uso do levamisol têm sido relatadas em animais tratados por diferentes métodos. Estas alterações incluem bradicardia (Montgomery e Pidgeon, 1986) e APCs em cães após emprego por via oral (Hoskins, 1983), conforme observado no presente estudo; bradicardia, taquicardia, complexos ventriculares prematuros (VPC), BAV e fibrilação ventricular em ratos, após uso intravenoso, corroborando este estudo, onde se observou ocorrência de BAV I e II após administração do levamisol por via oral (Onuaguluchi e Igbo, 1990).

Nogueira et al. (2003) relatam em cães a ocorrência de VPCs em cães tratados com levamisol em diferentes dosagens, pela via subcutânea, não observando, no entanto, alterações quando as mesmas doses foram utilizadas por via oral. Em contrapartida, neste estudo, baixas doses utilizadas por via oral foram capazes de alterar o ritmo cardíaco, demonstrando que os gatos provavelmente apresentem maior sensibilidade ao fármaco por via oral que os cães.

\section{CONCLUSÕES}

O levamisol, na dose utilizada, foi capaz de promover sinais de intoxicação, tais como sialórréia, midríase, vômitos e arritmias cardíacas, devendo-se ter cautela ao utilizá-lo em gatos. Não foram constatadas alterações do eritrograma, função hepática e proteínas séricas em gatos; entretanto, a diminuição significativa dos linfócitos após o tratamento com o fármaco, sugere a necessidade de novos estudos que avaliem os efeitos do mesmo sobre a função dos linfócitos e imunoglobulinas em gatos.

\section{NOTA INFORMATIVA}

A pesquisa foi aprovada pelo Comitê de Ética em Pesquisa da instituição de origem, protocolada sob o número 010/07.

\section{REFERÊNCIAS}

ALLEN, D.G.; DOWLING, P.M.; SMITH, D.A.

Handbook of veterinary drugs. 3.ed.

Philadelphia: Lippincott Williams \& Wilkins, 2005. $1111 \mathrm{p}$.

ALMEIDA, M.A.O.; AYRES, M.C.C. Considerações gerais sobre os anti-helmínticos. In: SPINOSA, H.S.; GÓRNIAK, S.L.; BERNARDI, M.M.

Farmacologia aplicada à medicina veterinária. 4.ed. Rio de Janeiro : Guanabara Koogan, 2006, Cap. 43, p.459-466. 
ANDRADE, S.F.; SANTARÉM, V.A.

Endoparasiticidas e ectoparasiticidas. In: ANDRADE, S.F. Manual de terapêutica veterinária. 2.ed. São Paulo : Roca, 2002, Cap.18, p.437- 476.

BALDANI, L.A.; SOUSA, R.V.; MIGUEL, A.G. [1999]. Farmacologia dos principais antiparasitários de uso na medicina veterinária. Boletim informativo da UFLA, Lavras, n.42, 1999.

Disponível em:

<http://www.editora.ufla.br/boletim/pdf/bol_42.pdf> Acesso em: 01/05/2007.

BOOTH, N.H.; McDONALD, L.M. Farmacologia e terapêutica em veterinária. 6.ed. Rio de Janeiro : Guanabara Koogan, 1992. 997 p.

CANAL, I.H.; CANAL, R.B.; DIDIANO, J.M.

Levamisol - Vermífugo e imuno modulador: antigo e eficaz - Uma revisão bibliográfica e indicações de uso. Revista Electrónica de Veterinaria REDVET, v.5, n.7, 2004. Disponível em:

$<$ http://www.veterinaria.org/revistas/redvet/n070704 B.htm>. Acesso em: 29/03/2007.

HOSKINS, J.D. Arrhythmia associated with levamisole heartworm therapy in a dog. Journal of the American Veterinary Medical Association, v.183, n.3, p.330-331, 1983.

JAIN, N.C. Essentials of Veterinary Hematology. Philadelphia : Lea \& Febiger, 1993. 417 p.

KANEKO, J.J.; HARVEY, J.W.; BRUSS, M.L.

Clinical biochemistry of domestic animals. $5 \mathrm{ed}$. San Diego : Academic Press, 1997. 932 p.

MEYER, D.J.; HARVEY, J.W. Veterinary

laboratory medicine, interpretation \& diagnosis.

2 ed. Philadelphia : W. B. Saunders, 1998. 373 p.
MONTGOMERY, R.D.; PIDGEON, G.L. Levamisole toxicosis in a dog. Journal of the American Veterinary Medical Association, v.189, n.6, p.684-685, 1986.

MORRISON, D.F. Multivariate Statistical

Methods. São Paulo : Mc Graw-Hill, 1990. 450 p.

NOGUEIRA, R.B.; SAKATE, M.; MATSUBARA, B.B.; GONÇALVES, R.S.; ROSA, G.J.M. Evaluation of cardiac rhythm in dogs treated with levamisole hydrochloride using 24-hour ambulatory. Journal of Veterinary Cardiology, v.5, n.2, p.29-32, 2003.

ONUAGULUCHI, G.; IGBO, I.N.

Electrocardiographic changes induced by levamisole hydrochloride in the rat. Archives Internationales de Pharmacodynamie et de Thérapie, v.305, p.55-62, 1990.

ROCHETT, J. Treating the inflamed mouth. In: WORLD SMALL ANIMAL VETERINARY WORLD CONGRESS, 26., 2001. Prcoceedings...

Vancouver. Disponível em:

<http://www.vin.com/VINDBPub/SearchPB/Proceedi ngs/PR05000/PR00067.htm>. Acesso em: 28/04/2007.

SCOTT, D. W.; MILLER, W. H.; GRIFFIN, C. E. Immune-mediated disorders. In: Muller \& Kirk's small animal dermatology. 6 ed. Philadelphia: W. B. Saunders Company, 2001, Cap.9, p.667-779.

SOUSA, M.G.; TALIER, I.C.; JORGE, A.T.B. et al. Reação farmacodérmica decorrente do uso do levamisol: relato de caso. Arquivo Brasileiro de Medicina Veterinária e Zootecnia, v.57, supl.2, p.154-157, 2005. 